\title{
Salience and learning rate in concept identification
}

BRUCE D. DOWNING ${ }^{1}$

UNIVERSITY OF COLORADO

Learning rate parameter estimates were computed for the Bower and Trabasso (1964) concept identification model on the basis of a cue salience measure. The estimates were employed in the model to generate theoretical learning curves which were compared with curves obtained from two types of concept identification tasks.

The Bower \& Trabasso (1964) model of concept identification (CI) has been fairly successful as a description of error probabilities in a two-choice task (e.g., Suppes \& Ginsberg, 1963; Bower \& Trabasso, 1964). Particularly the properties of stationarity and independence of presolution response probabilities are usually found in simple two-choice experiments employing binary dimensions. Stationarity and independence are interpreted as evidence of all-or-none learning.

One assumption of the Bower and Trabasso model is that the rate of learning depends on the salience or attention value of the relevant cues as compared to the salience of all cues. Bower \& Trabasso (1964) supported this assumption by providing adequate predictions of $\mathrm{CI}$ rates based upon the added weights of cues. Their technique involved the estimation of the CI rate parameter, $C$, when certain cues were relevant and redundant from the observed rates when each of the cues alone was relevant. In other words, they estimated the salience of cues from learning rates in order to predict learning rates. Another approach would be to measure the salience of cues in a completely different task, and then apply this salience measure to predict learning rate in a CI task. Testing such a measure was the main purpose of the experiment reported below.

A second purpose of the study was to determine if the model could be extended to a simple two-choice CI task with more than two levels per dimension. Method

A total of 26 paid adult Ss were given two tasks to perform in a single experimental session. They were given a search and identification (S\&I) task from which the estimates of cue salience were taken. Next, they were given a two-choice CI task in which half of the Ss were presented with stimuli having three binary dimensions. Stimuli for the remaining Ss were described by three dimensions with four levels per dimension.

The S\&I task consisted of the simultaneous presentation of all stimuli that were to be presented in the CI task. The stimuli were cards from the Wiscon- sin Card Sort task with color, shape, and number dimensions. The Ss were instructed to find a particular card that the $\mathrm{E}$ was thinking of by asking questions that could be answered "yes" or "no" about the characteristics of the cards. Actually, there was no predetermined card but the E answered "no" until the last level of each dimension was interrogated. The levels of all dimensions were inversely ranked according to the order in which they were interrogated so that the first level interrogated received the rank with the highest numerical value. Each $S$ solved four S\&I problems and the resulting ranks were summed in a manner that provided two estimates $\left(S_{1}\right.$ and $\left.S_{2}\right)$ of the salience of cues. These estimates (described below) were then used to replace $c$ in the Bower and Trabasso equation for cumulative probability of total errors, $P(T \leq k)=(1-c)^{k}$. The resulting probability distributions (for $S_{1}, S_{2}$, and c) were compared with the curves obtained in the CI tasks. The Bower and Trabasso parameter $c$ was estimated from the mean total errors in the CI task $(c=1 / \bar{T})$.

In the $\mathrm{CI}$ task with binary dimensions, stimulus cards that were presented simultaneously in the S\&I situation were presented successively by means of a slide projector and Ss were instructed to classify each stimulus by pressing one of two buttons. Immediate feedback as to whether or not the response was correct was given. The CI task with four levels per dimension was similar to the two-level problem except that Ss were instructed to find a single relevant level and press Button 1 when it was present, Button 2 when it was not present. The number dimension was relevant for the two-level case and one particular number was relevant for the four-level problem.

The first search and identification estimate $\left(S_{1}\right)$ of the salience of relevant cues was computed differently depending on the number of levels per dimension in the problem. For the two-level problem, it was assumed that $\mathrm{Ss}$ attend to entire dimensions and not to specific levels of a dimension. The estimate is computed from the ranks obtained from the questions asked in the S\&I task about each dimension, without ranking the levels separately. Therefore, $s_{1}$ for the two-level problem is the sum of the ranks assigned to the relevant dimension over the sum of the ranks of all dimensions.

It is assumed for the four-level problem that Ss are testing spectfic levels in an effort to determine the single relevant level. Therefore, the parameter estimate $S_{1}$ is the sum of the ranks assigned to the 
Table 1

Salience estimates, maximum deviations and mean absolute deviations of predicted from observed points

\begin{tabular}{lcccccc} 
& \multicolumn{3}{c}{ Two Levels } & \multicolumn{3}{c}{ Four Levels } \\
\hline & $\mathrm{S}_{1}$ & $\mathrm{~S}_{2}$ & $\mathrm{C}$ & $\mathrm{S}_{1}$ & $\mathrm{~S}_{2}$ & $\mathrm{C}$ \\
Value & .118 & .215 & .173 & .061 & .087 & .116 \\
Max. (D) & .243 & .236 & .148 & .311 & .113 & .153 \\
Mean (D) & .124 & .125 & .087 & .180 & .086 & .082 \\
\hline
\end{tabular}

single relevant level over the sum of the ranks of all of the individual levels regardless of the dimension.

The second S\&I estimate $\left(\mathrm{S}_{2}\right)$ of the salience of relevant cues is based upon a common assumption for both the two- and four-level problems. The assumption is that Ss will first test all levels of one dimension. If the relevant cue is not discovered, $S$ will then test all levels of another dimension, and so on. Based upon these assumptions $S_{2}$ is taken as the average salience proportion of the levels on the relevant dimension. That is, a salience proportion which is the sum of ranks of a particular level over the sum of ranks of all levels is computed for each level on the relevant dimension. The average of these proportions is $S_{2}$. Therefore, $S_{2}$ is not a measure of the salience of a particular dimension or level as with $S_{1}$, but is the average salience of all levels on a single relevant dimension.

\section{Resulis and Discussion}

The observed and predicted cumulative probability curves, $P(T \leq k)$, for the two-level and four-level problems were computed. Table 1 presents the salience estimates used to generate the predicted probability points, the maximum absolute difference between each predicted and observed point (for each value of $k$ ), and the mean absolute difference. None of the maximum absolute differences is significant according to the Kolmogorov-Smirnov one sample test (maximum D for the four-level case, $S_{1}$ estimate, is significant at the .15 level).

The results in Table 1 indicate that in the two-level case $S_{1}$ underestimates the observed points as much as $\mathrm{S}_{2}$ overestimates the same points. The cestimate taken from the Cl data provides the best fit to the two-level problem. It is interesting to note that although Bower \& Trabasso (1964) state that the model will not hold for more than two levels per dimension, the equation for the cumulative total errors provides a reasonable fit with the $S_{2}$ and cestimates of learning rate for the four-level task.

The interpretation of the results in terms of the assumptions behind the $S_{1}$ and $S_{2}$ estimates is not entirely clear. Neither the assumption that Ss attend to dimensions nor the assumption that they attend to levels is supported. Verbal reports of the Ss after the experiment indicated that in the two-level situation four Ss tested the relevancy of dimensions by responding to both levels of each dimension until correct, four Ss responded to individual levels across dimensions, and the remaining Ss combined these strategies. The use of more than one response mode may account for the data falling between the values predicted on the basis of pure strategies $\left(S_{1}\right.$ and $\left.S_{2}\right)$.

The results for the four-level problem indicate that the estimate based on the average salience of the levels on the relevant dimension $\left(S_{2}\right)$ provides a reasonably good fit to the data. The postexperimental verbal reports of the Ss revealed that nine of the $13 \mathrm{Ss}$ pressed the second button and systematically tested for relevance the levels of a single dimension before testing the levels of other dimensions. The average salience of the levels on the relevant dimension would, therefore, indicate the order of testing and those levels relative to the levels of other dimensions, and this is what the $\mathrm{S}_{2}$ estimate measures.

Stationarity and independence analyses were performed on the presolution data using the methods described by Atkinson, Bower, \& Crothers (1965). The analyses for the two-level problem supported stationarity $\left(X^{2}=5.56, \mathrm{df}=8, p>.5\right)$ and independence $\left(x^{2}=3.59, \mathrm{df}=1, \mathrm{p}>.05\right)$. The same analyses for the four-level presolution data indicated lack of stationarity $\left(X^{2}=21.74, \mathrm{df}=12, \mathrm{p}<.05\right)$ and lack of independence $\left(x^{2}=10.62, \mathrm{df}=1, p<.005\right)$. Therefore, the all-or-none learning hypothesis is supported by the two-level data but not the four-level data. This finding supports the limitations placed by Bower and Trabasso upon their model.

In conclusion, it would seem that the measurement of salience through the S\&I task is promising but not definitive. Of course, it is quite possible that factors other than salience influence the rate of learning in a CI task. The possibility of employing strategies such as Ss reported in the four-level problem may explain some of the difficulty found when trying to apply the Bower and Trabasso model beyond the two-choice CI task with binary dimensions.

\section{References}

ATKINSON, R. C., BOWER, G. H., \& CROTHERS, E. J. An Introduction to Mathematical Learning Theory. New York: John Wiley \& Sons, 1965.

BOWER, G. H., \& TRABASSO, T. Concept Identification in R. C. Atkinson (Ed.), Studies in Mathematical Psychology, Stanford University Press, 1964.

SUPPES, P., \& GINSBERG, R. A fundamental property of all-or-none models, binomial distribution of responses prior to conditioning, with application to concept formation in children. Psychol. Rev., 1963, $70,139-161$.

Note

1. Now at Kent State University, Kent, Ohio 